\title{
Presentación/Editorial Presentation/Editorial
}

\section{Cabello/Carceller}

Sin Objeto. Arte, Investigación, Políticas aparece como una revista digital de periodicidad anual. Surge de la necesidad de vincular la producción artística que se realiza en la actualidad con los trabajos teóricos que creemos deberían ayudar a construir un pensamiento transdisciplinar, consciente de los contextos en los que las manifestaciones artísticas son actualmente producidas y recibidas.

Partimos de la necesidad de reestructurar los espacios de las representaciones incorporando dimensiones conceptuales, sociopolíticas y una temporalidad transversal en las propuestas.

Sin Objeto pretende poner en diálogo a investigadores académicos con investigadores no necesariamente vinculados al sistema universitario y artistas en activo, para pensar en torno a problemáticas comunes. En este sentido se plantea como una plataforma a través de la cual acercar posiciones e intentar vincular la realidad universitaria española a la praxis artística, superando escisiones obsoletas y reconduciendo las diferencias artificiosas entre áreas de conocimiento todavía organizadas según una tradición cognitiva decimonónica, ya inoperativa.

Como revista surge en un momento en el que la desmaterialización de las prácticas es un hecho que acompaña a aquellas más visibles, e incluso salpica a muchas que aparentemente la negarían en su presentación. Así, los “objetos” a través de los que se expresan muchos proyectos no pueden ya concebirse aislados de las subjetividades que los proponen y de los espacios en los que estas subjetividades cohabitan. Es pues un lugar para el diálogo, pero en un sentido agonístico —en palabras de Laclau y Mouffe-, es decir, sin eludir el disenso.

La revista se estructurará en varias secciones. Cada número incorporará un dossier temático en el que diferentes teóricos analizarán el estado de la cuestión. Como parte del dossier podrán incluirse también traducciones de textos no publicados en castellano que por su importancia se consideren relevantes para ampliar la perspectiva del tema elegido. A partir del número 1, también se incluirán aquellos 
artículos originales que hayan sido enviados por quienes respondan a la convocatoria pública, una vez superada la correspondiente revisión por pares. Los artículos que estudien el tema propuesto se publicarán como parte del dossier, los que se alejen de él lo harán en una sección de investigación, abierta también a capítulos de tesis no publicados y primeros resultados de investigaciones en curso. Además del dossier y de la sección de investigación, se publicarán una conversación artista-teórico/a y se invitará a artistas a realizar proyectos ex profeso. La revista incluirá también una sección de recensiones de publicaciones y/o crítica de exposiciones, proyectos participativos...

Sin Objeto es una revista publicada por la Universidad de Castilla-La Mancha cuyo número 0 se inscribe dentro del proyecto de investigación "Creación y estudios de las CAAC (Colecciones y Archivos de Arte Contemporáneo) de Cuenca como modelo metodológico para una investigación de excelencia en Bellas Artes" (HAR2013-48604-C2-1-P).

\section{Coleccionar lo inmaterial}

Iniciamos la revista Sin Objeto con un número que, bajo el título Coleccionar lo inmaterial, analiza algunas de las problemáticas a las que se enfrentan actualmente las instituciones museísticas y el mercado del arte. A priori una paradoja, la idea de coleccionar aquello que es difícilmente aprehensible (performances, proposiciones, proyectos realizados con materiales efímeros o que se pueden considerar site-specific y estar destinados a desaparecer, etc.) plantea un reto importante al museo actual; pero también a los distintos agentes implicados en la producción, presentación y/o conservación artística.

En esta ocasión hemos contado para el dossier con la colaboración de una persona que posee una larga trayectoria en la gestión y dirección de museos y colecciones, Glòria Picazo, quien en su contribución "Nuevos retos para las colecciones de arte contemporáneo" analiza y plantea las alternativas posibles en una situación en la que lo efímero debe encontrar forzosamente su espacio en las colecciones públicas. Las problemáticas que genera al coleccionismo el auge de la performance son revisadas por Juan Albarrán, profesor de la Universidad Autónoma de Madrid, a través del trabajo del artista Tino Sehgal en su artículo "Sehgal no invita a la lógica. Performance, experiencia y economía inmaterial en Tino Sehgal". Sehgal, ganador del León de Oro en la 55 Bienal de Venecia en 2013, centra su práctica artística en cuestionar la manera en la que la performance es coleccionada, o quizá sería mejor decir coleccionable, y en su relación con las instituciones artísticas. Por su parte José Ramón Alcalá, catedrático de la Universidad de Castilla-La Mancha, en su artículo "Musealización de las prác- 
ticas artísticas contemporáneas. Colecciones y archivos: nuevas estrategias para su gestión, promoción y difusión" reconsidera, utilizando como caso de estudio las colecciones universitarias de la Facultad de Bellas Artes de Cuenca y de la UCLM, Colecciones y Archivos de Arte Contemporáneo (CAAC), el modelo museístico actual y las problemáticas que le ha planteado el surgimiento del conocido como Media art. Para esta ocasión hemos seleccionado también las contribuciones científicas de Renée van de Vall, catedrática adscrita a la Universidad de Maastricht, "Documenting dilemmas. On the relevance of ethically ambiguous cases" y de Robin K. Williams, Universidad de Texas, "A Mode of Translation: Joan Jonas's Performance Installations". La profesora van de Vall revisa la necesidad ética de ir analizando "caso a caso" para poder enfrentarse responsablemente a los problemas que van surgiendo en el ejercicio de la conservación de los trabajos artísticos. Por su parte Williams utiliza a Joan Jonas, artista pionera de la performance, como punto de partida para reflexionar en torno a las complicaciones que afloran en aquellos proyectos que se nutren de múltiples elementos performativos y explora cómo intentan ser resueltos por la propia artista.

En la sección Investigación Maite Aldaz analiza a través de la figura del conocido artista Daniel Buren las problemáticas que plantea al coleccionismo institucional la práctica del site-specific. Por otro lado, en el apartado Conversaciones, contamos con la participación de la artista Eulàlia Valldosera y de la profesora de la Facultad de Bellas Artes de Cuenca y comisaria de exposiciones Amparo Lozano. Valldosera, una artista que comenzara su carrera a principios de los años 90 , ha mantenido desde sus inicios una práctica artística performativa y desmaterializada, inscrita sin embargo dentro del circuito de galerías y del coleccionismo público y privado.

El número 0 de Sin Objeto se completa con los proyectos específicos realizados para la revista por los artistas Elena Bajo ("Reactivation of Memory: The Pleiades") y Marco Godoy ("Que lo que está colgado toque el suelo") y con la recensión de Andrés Foglia sobre el libro de Boris Groys Arte en flujo. Ensayos sobre la evanescencia del presente.

\section{Cita Recomendada}

Cabello Carceller (2017). Presentación/Editorial. Sin Objeto, 00, 07-11. 


\section{Biografía}

\section{Cabello/Carceller}

Universidad de Castilla-La Mancha

helena.cabello@uclm.es

ana.carceller@uclm.es

Cabello/Carceller es un equipo artístico formado por Helena Cabello y Ana Carceller. Como artistas han participado en múltiples exposiciones en centros de arte y museos nacionales e internacionales: 56 Bienal de Venecia, Bienal de Bucarest BB4, Brooklyn Museum de Nueva York, Casino Luxembourg o MNCARS. Entre sus últimas exposiciones individuales destacan las presentadas en CA2M y MARCO de Vigo, IVAM de Valencia, CCEMX o Matadero Madrid.

Sus publicaciones recientes incluyen los libros/catálogos: Borrador para una trama en curso, Lost in Transition_un poema performativo y Distrito Federal: La búsqueda del archivo. Ficción $n^{\circ} 9$.

Helena Cabello es doctora en Bellas Artes por la Universidad de Vigo y actualmente profesora contratada doctora (acreditada a titular de universidad) en el Departamento de Arte de la Facultad de Bellas Artes de Cuenca, Universidad de Castilla-La Mancha. Ha impartido docencia en la Universidad Europea de Madrid, donde dirigió el Área de Arte, y en la Universidad Miguel Hernández de Elche. Además ha colaborado en diversos programas de Máster y postgrado en distintas universidades y centros de investigación, entre ellos el Máster en Historia del Arte Contemporáneo y Cultura Visual (MNCARS/ UCM/ UAM), el Máster Oficial en Producción Artística de la UPV o el Máster en Educación Artística (Facultad de BBAA de la Universidad de Lisboa) y el Máster en Estudios Curatoriales (Universidad de Lisboa/Fundaçao Calouste Gulbenkian). Recientemente ha sido invitada como Distinguished Professor en el Haverford College de Philadelphia (EE.UU.). Ha participado en diversos proyectos de investigación relacionados con las prácticas artísticas contemporáneas, los feminismos y las teorías de género y ha sido investigadora principal en el proyecto de investigación TestMadrid. Espacio experimental de intervención artística (UEM/Ayuntamiento de Madrid).

Ana Carceller es doctora en Bellas Artes por la Universidad de Vigo y actualmente profesora contratada doctora (acreditada a titular de universidad) en el Departamento de Arte de la Facultad de Bellas Artes de Cuenca, Universidad de Castilla-La Mancha. Ha impartido docencia en la Universidad Europea de Madrid, en la Universidad Miguel Hernández de Elche y en la Universidad de Vigo. Además ha colaborado en diversos programas de Máster y postgrado en distintas universidades y centros de investigación, entre ellos el Máster en Historia del Arte 
Contemporáneo y Cultura Visual (MNCARS/ UCM/ UAM), el Máster Oficial en Producción Artística de la UPV o el Máster en Educación Artística (Facultad de BBAA de la Universidad de Lisboa) y el Máster en Estudios Curatoriales (Universidad de Lisboa/Fundaçao Calouste Gulbenkian). Recientemente ha sido invitada como Distinguished Professor en el Haverford College de Philadelphia (EE.UU.). Ha participado en diversos proyectos de investigación relacionados con las prácticas artísticas contemporáneas, los feminismos y las teorías de género. Entre los más recientes, destaca su participación como investigadora en el proyecto de Excelencia I+D Creación y Estudio de las CAAC de Cuenca como modelo metodológico para una investigación de Excelencia en Bellas Artes. 